ARTICLE

\title{
The epidemiology of Plasmodium vivax among adults in the Democratic Republic of the Congo
}

\author{
Nicholas F. Brazeau1,2凶${ }^{1,}$, Cedar L. Mitchell', Andrew P. Morgan²,3, Molly Deutsch-Feldman', \\ Oliver John Watson (10 4, Kyaw L. Thwai ${ }^{1}$, Pere Gelabert (1) ${ }^{5,6}$, Lucy van Dorp (1) ${ }^{5}$, Corinna Y. Keeler (1) ${ }^{1}$, \\ Andreea Waltmann77, Michael Emch ${ }^{8}$, Valerie Gartner (1) 9 , Ben Redelings9 ${ }^{9}$, Gregory A. Wray (1) 9,10, \\ Melchior K. Mwandagalirwa ${ }^{11}$, Antoinette K. Tshefu"1, Joris L. Likwela12, Jessie K. Edwards (1) ${ }^{1}$, Robert Verity (D) ${ }^{4}$, \\ Jonathan B. Parr ${ }^{13,14}$, Steven R. Meshnick ${ }^{1,15}$ \& Jonathan J. Juliano 1,13,14,15
}

Reports of $P$. vivax infections among Duffy-negative hosts have accumulated throughout subSaharan Africa. Despite this growing body of evidence, no nationally representative epidemiological surveys of $P$. vivax in sub-Saharan Africa have been performed. To overcome this gap in knowledge, we screened over 17,000 adults in the Democratic Republic of the Congo (DRC) for P. vivax using samples from the 2013-2014 Demographic Health Survey. Overall, we found a $2.97 \%$ ( $95 \% \mathrm{Cl}: 2.28 \%, 3.65 \%)$ prevalence of $P$. vivax infections across the DRC. Infections were associated with few risk-factors and demonstrated a relatively flat distribution of prevalence across space with focal regions of relatively higher prevalence in the north and northeast. Mitochondrial genomes suggested that DRC $P$. vivax were distinct from circulating non-human ape strains and an ancestral European $P$. vivax strain, and instead may be part of a separate contemporary clade. Our findings suggest $P$. vivax is diffusely spread across the DRC at a low prevalence, which may be associated with long-term carriage of low parasitemia, frequent relapses, or a general pool of infections with limited forward propagation.

\footnotetext{
${ }^{1}$ Department of Epidemiology, Gillings School of Global Public Health, University of North Carolina, Chapel Hill, NC, USA. ${ }^{2}$ Medical Scientist Training Program, School of Medicine, University of North Carolina, Chapel Hill, NC, USA. ${ }^{3}$ Department of Bioinformatics \& Computational Biology, University of North Carolina, Chapel Hill, NC, USA. ${ }^{4}$ Medical Research Council Centre for Outbreak Analysis and Modelling, Department of Infectious Disease Epidemiology, Imperial College London, London, UK. ${ }^{5}$ UCL Genetics Institute, University College London, London, UK. ${ }^{6}$ Department of Evolutionary Anthropology, University of Vienna, Vienna, Austria. ${ }^{7}$ Institute for Global Health and Infectious Diseases, School of Medicine, University of North Carolina, Chapel Hill, NC, USA. ${ }^{8}$ Department of Geography, University of North Carolina, Chapel Hill, NC, USA. ${ }^{9}$ Department of Biology, Duke University, Durham, NC, USA. ${ }^{10}$ Duke Center for Genomic and Computational Biology, Durham, NC, USA. ${ }^{11}$ Kinshasa School of Public Health, Kinshasa, Democratic Republic of the Congo. ${ }^{12}$ Programme National de la Lutte Contre le Paludisme, Kinshasa, Democratic Republic of Congo. ${ }^{13}$ Division of Infectious Diseases, School of Medicine, University of North Carolina, Chapel Hill, NC, USA. ${ }^{14}$ Curriculum in Genetics and Molecular Biology, School of Medicine, University of North Carolina, Chapel Hill, NC, USA. ${ }^{15}$ These authors contributed equally: Steven R. Meshnick, Jonathan J. Juliano. ${ }^{凶}$ email: nbrazeau@med.unc.edu
} 
P lasmodium vivax is the most prevalent malaria-causing parasite in many locations outside of Africa, accounting for $\sim 6.4$ million cases in $2019^{1}$. The relative absence of $P$. vivax in Africa has long been attributed to the high prevalence of the Duffy-negative phenotype throughout most of sub-Saharan Africa (SSA) ${ }^{2-4}$. However, recent evidence has demonstrated that $P$. vivax infections are occurring throughout SSA among both Duffy-positive and Duffy-negative hosts ${ }^{5}$. Although these $P$. vivax infections have been associated with clinical cases, the distribution and extent of asymptomatic versus symptomatic disease in SSA remains unclear ${ }^{5-7}$.

Despite growing concern, no studies have systematically evaluated the burden, risk factors, spatial distribution, or origins of these SSA P. vivax infections. This lack of research is problematic as resources have begun to be directed towards diagnosing and addressing SSA $P$. vivax. If $P$. vivax were returning or reemerging in SSA as a new epidemic, it would have the potential to undermine years of malaria control and elimination efforts. To address this critical gap in knowledge, we used samples from the Democratic Republic of the Congo (DRC) 2013-2014 Demographic Health Survey (DHS) to screen a nationally representative population of over 17,000 adults for $P$. vivax. Surveys from the DHS program are community-based and are expected to contain mostly healthy, asymptomatic participants. The DRC is situated in the center of SSA and is the largest country by geographic size. Moreover, previous work has indicated that the DRC is a watershed region that links East and West Africa malaria ${ }^{8,9}$. As a result, findings from the DRC are highly relevant for contextualizing vivax malaria in SSA.

Using this nationally representative survey, we provide a national level estimate of $P$. vivax prevalence, associated risk factors, and the geographical distribution of cases across the DRC. In addition, we use mitochondrial genomes to identify the potential origins of these infections. By coupling a nationally representative, spatially rich dataset with robust risk factor and spatial analysis, we advance efforts to uncover the hidden distribution of $P$. vivax in SSA.

\section{Results}

Study population and molecular validation. $P$. vivax infections were first identified using quantitative PCR (qPCR) and then confirmed with a nested-PCR assay. Our $P$. vivax $\mathrm{qPCR}$ assay achieved an analytical sensitivity of $94 \%$ and analytical specificity of $100 \%$ (zero false positive calls) when at least $1.25 \times 10^{-7} \mathrm{ng} / \mu \mathrm{L}$ of $18 \mathrm{~S}$ rRNA plasmid (equivalent to approximately the number of copies of $18 \mathrm{~S}$ rRNA in 6 genomes $/ \mu \mathrm{L}$ ) was present. No off-target amplification was observed when the qPCR assay was challenged with highly concentrated DNA templates from other Plasmodium species (Supplementary Fig. 1, Supplementary Table 2). Of the 17,972 samples that underwent screening for $P$. vivax infection, 579 were positive by qPCR. Among the $579 P$. vivax qPCRpositive samples, 534 were confirmed by nested-PCR (92.22\%), with strong agreement between the initial and reflex confirmatory assays (Cohen's $\mathscr{K}=0.80, p<0.05$ ). All samples selected for Duffy-Genotyping validation had concordant HRM-qPCR and Sanger sequencing results, except for one sample that failed genotyping (Supplementary Text: Duffy-Genotyping). We restricted our prevalence estimates to the $467 P$. vivax infections that were confirmed by both qPCR and reflex nested-PCR $\left(n_{\text {weighted: }}: 459.18,95 \% \mathrm{CI}_{\text {weighted }}: 346.54,571.82\right)$ and were among the 15,574 adults included in our study ( $n_{\text {weighted }}: 15,490.20,95 \%$ $\mathrm{CI}_{\text {weighted: }}$ 14,060.60, 16,919.80; Fig. 1).

Prevalence of $P$. vivax and descriptive statistics among adults in the DRC. The national weighted prevalence of $P$. vivax among adults was $2.96 \%\left(95 \% \mathrm{CI}_{\text {weighted }}: 2.28,3.65 \%\right)$. Among the 489 clusters considered, 172 clusters were positive for $P$. vivax (range: 1-9 infections per cluster) with 64 clusters containing a single infection. When the DHS sampling weights were applied, $P$. vivax infection counts ranged from 0.15 to 30.63 among the 172 clusters. Given that DHS sampling weights are applied at the clusterlevel, $P$. vivax unweighted and weighted prevalences were the same and ranged from 0 to $46.15 \%$ with wide confidence intervals (Supplementary Fig. 2).

In contrast, we identified $5179 P$. falciparum infections ( $\left.n_{\text {weighted }}: 4651.94,95 \% \mathrm{CI}_{\text {weighted}}: 4121.93,5181.94\right)$ accounting for a weighted national prevalence of $30.03 \%$ (95\% $\mathrm{CI}_{\text {weighted: }}$ 27.87, 32.19\%). Overall, we identified $174 P$. falciparum-P. vivax co-infections that were reduced to 145.29 co-infections when DHS sampling weights were applied $\left(95 \% \mathrm{CI}_{\text {weighted }}\right.$ : 108.11, 182.48; Table 1). Individual characteristics differed by infection status and suggested differences in demographic and behavioral factors between those individuals infected with $P$. vivax or $P$. falciparum versus those not infected (Table 1).

Risk factors. In order to identify "who" was being infected by $P$. vivax, we performed a risk factor analysis that included common malaria exposures, or covariates. We also performed a risk factor analysis with $P$. falciparum as the outcome of interest for comparison. Risk factors were estimated using prevalence odds ratios (pORs) that were adjusted for confounding using inverse probability weights (IPWs). IPWs were calculated with a supervised machine learning approach: a spatially cross-validated super learner algorithm. However, the super learner candidate library was reduced to a regression model (i.e. linear regression or logistic regression for continuous versus categorical variables) in $9 / 11$ models in favor of convergence or better IPW stability (Supplementary Table 6). For most covariates, IPWs were relatively stable, with some imbalances in housing materials and distance from healthcare facilities, and to a lesser extent, in education, farming, and wealth, potentially indicating lingering issues in structural positivity or residual confounding (Supplementary Fig. 6; Supplementary Table 5). Similarly, for most covariates, IPWs resulted in a considerable decrease in the average correlation among risk factors as compared to unadjusted baseline correlations (mean foldreduction: 3.73, range: 1.10-7.37; Supplementary Fig. 7). However, for two risk factors: HIV status and precipitation, the maximum IPW-based correlation exceeded the maximum baseline correlation, which may indicate residual confounding.

When $P$. vivax was considered as the outcome of interest, higher-levels of precipitation were associated with reduced prevalence (IPW-pOR: 0.68, 95\% CI: 0.52, 0.91) while further distances from healthcare facilities were associated with increased prevalence (IPW-pOR: 2.07, 95\% CI: 1.24, 3.46; Fig. 2). In conducting a sensitivity analysis on the coding of net-use, we found that our primary exposure, insecticide-treated net (ITN) use, contained the null for both the unweighted (Supplementary Table 7) and IPW-approach (Fig. 2) but that the DHS longlasting insecticide net use variable only contained the null for the unweighted approach (OR: 0.75, 95\% CI: 0.55, 1.01). For the IPW-approach, lack of long-lasting insecticide net use was associated with increased $P$. vivax prevalence (IPW-pOR: 0.70, 95\% CI: 0.52, 0.96).

In contrast, when considering $P$. falciparum infections as the outcome of interest, several risk factors were associated with increased prevalence: lack of ITN use (IPW-pOR: 1.31, 95\% CI: $1.14,1.50$ ), temperature (IPW-pOR: $1.25,95 \%$ CI: $1.10,1.42$ ), lower levels of education (IPW-pOR: 1.43, 95\% CI: 1.24, 1.65), low levels of wealth (PW-pOR: 1.31, 95\% CI: 1.14, 1.51), traditional housing materials (PW-pOR: 1.36, 95\% CI: 1.09, 


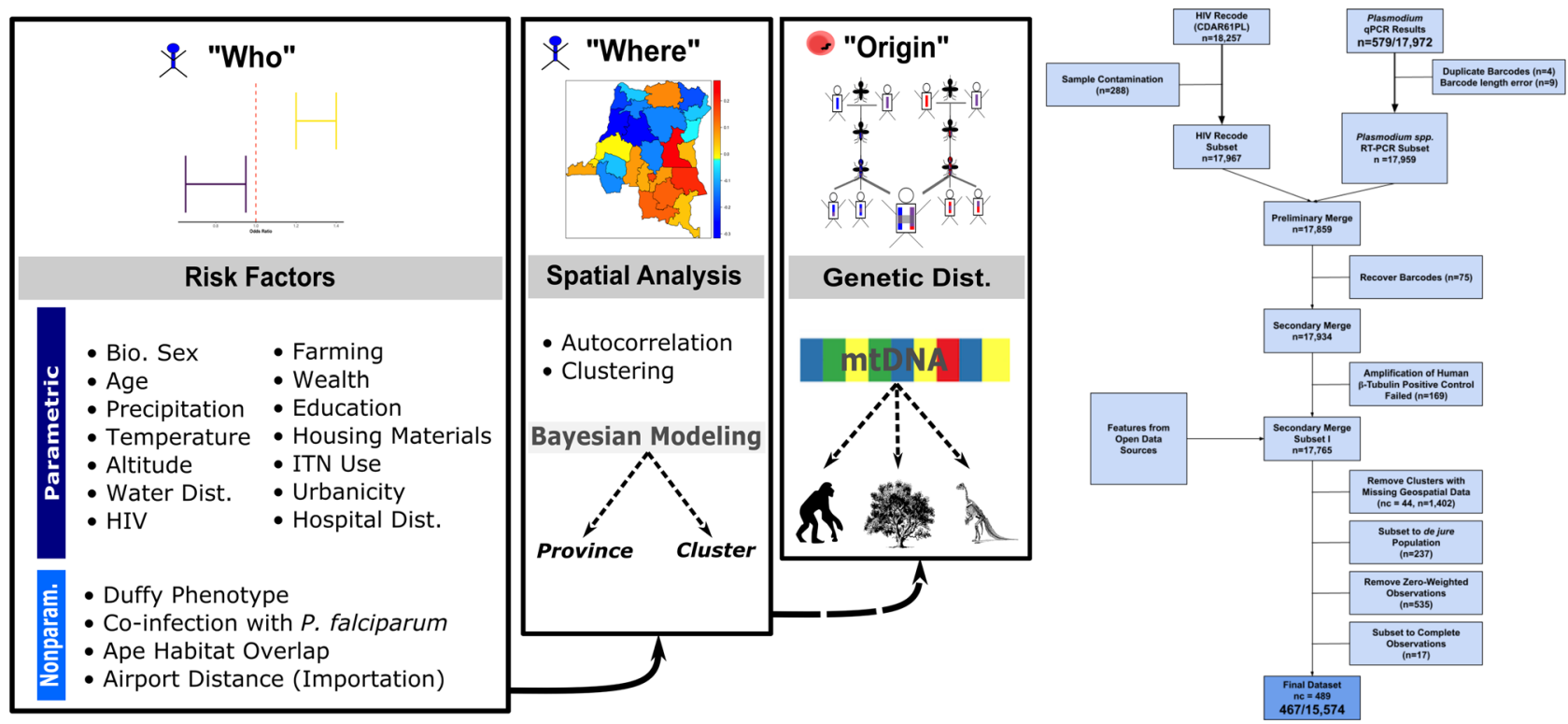

Fig. 1 Overview of study approach and inclusion. The conceptual diagram (left) describes the study approach to identify "who" was being infected by $P$. vivax, "where" P. vivax infections were occurring, and the potential source, or "origin", of these infections. Risk factors identified in the "who" analysis, were considered in the spatial models. Genetic distance analyses were restricted to the mitochondrial genome (mtDNA) and few DRC samples ( $n=3)$. As a result, we limited this analysis to three broad questions: are the DRC samples most similar to (1) non-human apes mitochondrial strains; (2) contemporary $P$. vivax mitochondrial strains; or (3) an ancestral P. vivax mitochondrial strain. The final data set (right) consisted of 467 P. vivax infections among 15,574 individuals across 489 clusters, prior to the application of the Demographic Health Survey (DHS) sampling weights. Specifically, of the 18,257 Demographic Health Survey (DHS) records that had a dried blood spot, 17,972 samples were successfully shipped to the University of North Carolina for processing. Of these 17,972 samples, a small portion were lost due to barcoding errors, while the remaining 17,934 (99.79\%) were successfully linked to the 2013-2014 DRC DHS survey. Of these 17,934 samples, 169 samples failed to amplify human beta-tubulin (i.e. positive control), 1402 individuals had missing geospatial data, 484 individuals were classified as de facto (visitors rather than household members), 535 individuals had zero-weighted DHS sampling weights, and 17 individuals had incomplete data and were excluded from analysis (further details in Supplementary Materials: Study Population and Data Sources). In total, our final dataset consisted of 15,574 individuals across 489 clusters. Similarly, although we identified a total of 579 P. vivax infections, 112 infections were lost during the aforementioned processing steps, which resulted in a final, unweighted count of $467 P$. vivax infections.

Table 1 Descriptive statistics of identified risk factors among individuals with $P$. vivax infections, $P$. falciparum infections, and those that are uninfected with DHS sampling weights applied: risk factor distributions appeared to differ by infection status.

\section{Covariate}

$N^{*}$

Vivax-Falciparum co-infection

Urban (\%)

Distance to healthcare facilities (far, \%)

HIV (positive, \%)

Biological sex (male, \%)

Farmer (\%)

Housing materials (traditional, \%)

Education (lower, \%)

ITN use (no, \%)

Wealth (poor, \%)

Precipitation (mm, SD)

Temperature (C, SD)

Altitude (m, SD)

Distance to water ( $m, S D)$

Age (years, SD)

Number of household members ( $N, S D)$

P. vivax infection
459.18
145.29
$195.75(42.63)$
$247.34(53.86)$
$8.09(1.76)$
$235.89(51.37)$
$202.64(44.13)$
$262.10(57.08)$
$181.79(39.59)$
$213.83(46.57)$
$168.12(36.61)$
$131.97(25.79)$
$25.98(1.99)$
$771.46(489.10)$
$6695.73(7745.45)$
$29.35(11.42)$
$6.57(3.08)$

P. falciparum infection

4651.94

$1573.16(33.82)$

$2524.99(54.28)$

$26.76(0.58)$

$2435.38(52.35)$

$2061.67(44.32)$

$3127.13(67.22)$

$1975.86(42.47)$

$2611.93(56.15)$

$2209.84(47.50)$

137.85 (24.97)

$26.75(1.35)$

$617.56(310.44)$

$7762.31(8096.52)$

$28.17(10.84)$

$6.67(3.08)$

Uninfected
$10,524.38$
-
$4445.27(42.24)$
$4645.82(44.14)$
$117.99(1.12)$
$4790.0(45.51)$
$4181.96(39.74)$
$5555.21(52.78)$
$4063.91(38.61)$
$5301.37(50.37)$
$4210.55(40.01)$
$138.33(24.70)$
$26.15(1.80)$
$749.62(473.77)$
$6364.54(7074.80)$
$30.39(10.97)$
$6.78(3.29)$

For dichotomized risk factors, the weighted counts and weighted percentages for each category are provided. For continuous risk factors, the mean and standard deviation (SD) are provided. $N$ number of individuals, $m m$ millimeters, $m$ meters, ITN insecticide-treated net.

${ }^{\star}$ Co-infections are included for both the $P$. vivax and $P$. falciparum marginal infection counts.

1.71), and being male (IPW-pOR: 1.31, 95\% CI: $1.20,1.43$ ). Additionally, three risk factors were associated with decreased prevalence: an urban setting (IPW-pOR: 0.70, 95\% CI: 0.56, 0.86), increasing altitude (IPW-pOR: 0.73, 95\% CI: 0.66, 0.80), and older age (IPW-pOR: 0.81, 95\% CI: 0.77, 0.86; Fig. 2).
Based on our post hoc power calculations for $P$. vivax, we were able to detect approximate pOR estimates of at least $1.55,1.36$, 1.31 with at least $80 \%$ power when the exposure probability was $10 \%, 25 \%$, and $50 \%$, respectively. In contrast, for P. falciparum, we were able to detect approximate pOR estimates of at least 1.18, 


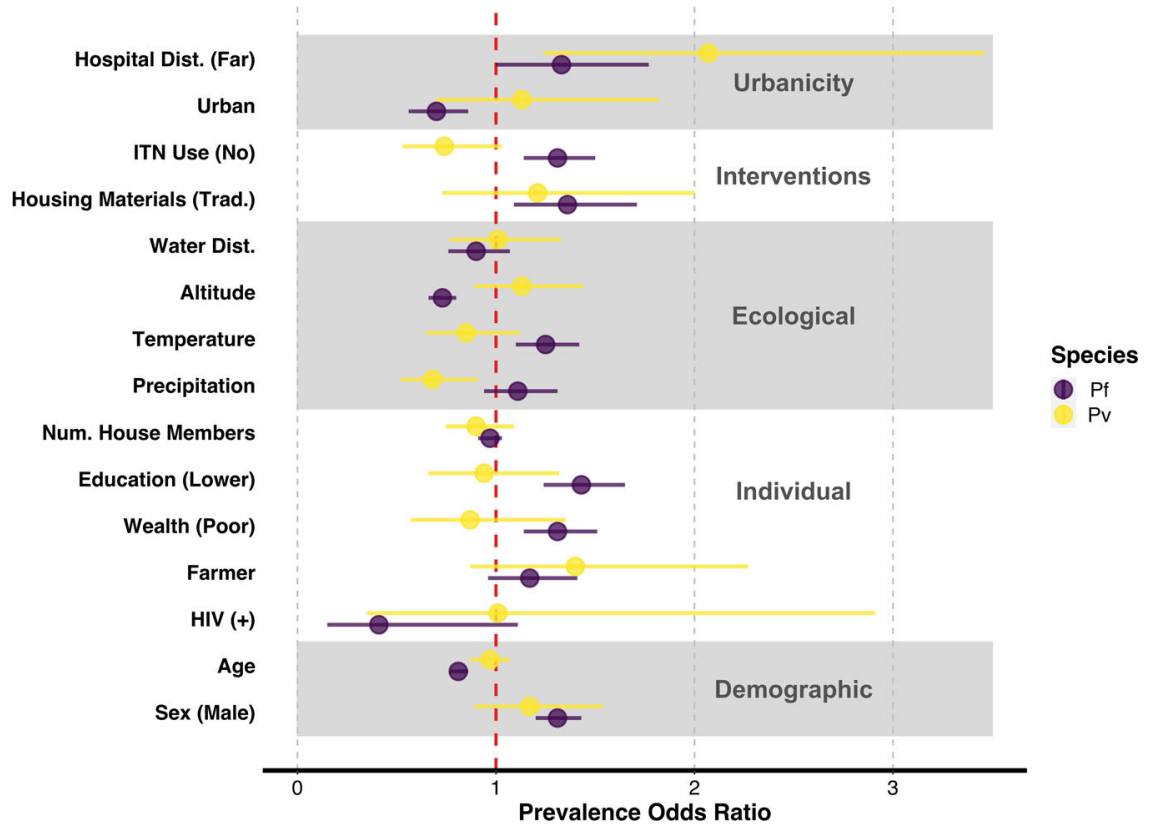

Fig. 2 Inverse probability weight adjusted prevalence odds ratios for expected malaria risk factors. Risk factor point estimates and associated $95 \%$ confidence intervals are displayed for $P$. vivax and $P$. falciparum, respectively. Risk factors associated with $P$. vivax infection included precipitation and distance from healthcare facilities, while risk factors were associated with $P$. falciparum infection, included: urbanicity, housing materials, ITN use, altitude, temperature, education, wealth, age, and biological sex. The unadjusted pORs effect estimates and confidence intervals as well as the IPW-pORs are provided in Supplementary Table 7 for reference. Hospital Dist distance to healthcare facilities, Water Dist. distance to water, Trad. traditional, Num. House Members number of household members, ITN insecticide-treated net.

$1.12,1.10$ with at least $80 \%$ power when the exposure probability was $10 \%, 25 \%$, and $50 \%$, respectively (Supplementary Fig. 10).

A subset of risk factors were evaluated with non-parametric approaches due to concerns of traditional risk-factor model assumption violations or data limitations. These risk factors included: the Duffy-negative phenotype, $P$. falciparum- $P$. vivax infection interference, overlap with non-human ape habitats, and proximities to airports, as a proxy for importation of $P$. vivax via airline travel. Among those individuals infected with $P$. vivax and included in our study, three hosts were genotyped as heterozygous (-33T:T/C) at the loci associated with Duffy-negative phenotype (Prevalence: $0.64 \%$, 95\% CI: $0.13,1.87 \%$ ). Given that only $P$. vivax infected individuals were genotyped at the Duffy antigen loci, the overall prevalence of putative Duffy-positive phenotype was not generalizable to the entire DRC. From our cross-species interference model that assumes independent acquisition of infections from a multinomial likelihood, we failed to find any evidence of interactions between $P$. falciparum- $P$. vivax co-infections (Fig. 3A). This result suggests that infection with $P$. falciparum does not inhibit or promote $P$. vivax infection and vice-versa. Similarly, using permutation testing, we did not find an association between non-human ape habitats and $P$. vivax cluster prevalence (one-sided $p>0.05$; Fig. $3 \mathrm{~B}$ ) nor a correlation between $P$. vivax prevalence and airport proximity (Fig. 3C).

Spatial distribution of $\boldsymbol{P}$. vivax. After identifying $P$. vivax risk factors, we wanted to identify "where" $P$. vivax infections were occurring. The spatial distribution of $P$. vivax was first assessed for disease clustering with 'SaTScan' and using measures of autocorrelation with Moran's I. We then modeled $P$. vivax prevalence across the DRC using Bayesian mixed spatial models that included risk factors that were previously identified as significant. Bayesian mixed spatial models were considered at two levels: (1) the province-level (areal model) and (2) the cluster-level (Gaussian spatial process model). Province-based spatial models are important for intervention-planning, as most interventions in the DRC are implemented at the province-level. However, clusterlevel models with Gaussian processes may be more representative of the intrinsic malaria distribution under the assumption of a continuous spatial process.

A large, significant cluster of $P$. vivax was detected in the northern provinces of the DRC using 'SaTScan' (Fig. 3D). Several smaller clusters were circumscript within the larger northern cluster and occurred within the north-central and north-eastern provinces. All clusters exhibited significantly elevated prevalence estimates relative to neighboring clusters with one-sided $p$ values $<0.05$.

When considering spatial autocorrelation, we found that the province-level showed a slight signal of structure for $P$. vivax prevalence (Moran's I: 0.16 ; one-sided $p=0.05$ ), but this structure did not hold at the cluster-level (Moran's I: 0.02; onesided $p>0.05)$. Among the $P$. vivax province-level models considered, means-fitted province prevalences ranged from 1.25 to $7.26 \%$ (Fig. $4 \mathrm{~A}$ ). Standard errors for the province prevalence estimates ranged from 0.24 to $1.16 \%$ (Supplementary Fig. 9B).

When we modeled the spatial distribution of $P$. vivax at the cluster-level, $P$. vivax predicted prevalence ranged from 0.39 to $4.40 \%$ across the DRC (Fig. $4 \mathrm{~B}$ ). The standard errors around the prevalence predictions ranged from 0.26 to $4.18 \%$ (Supplementary Fig. 9C). P. vivax prevalence predictions were less than the observed national prevalence in $\sim 99 \%$ of predicted cell locations.

Spatial model parameter posteriors and effective sampling sizes for both the province-level and cluster-level model are available in Supplementary Table 8 . Similarly, the raster surfaces for the precipitation and distance from healthcare facilities covariates are displayed in Supplementary Fig. 8.

P. vivax mitochondrial genetic distances. To identify the phylogeographic affinities of DRC $P$. vivax, we performed a simple genetic analysis comparing the mitochondrial genome (mtDNA) 


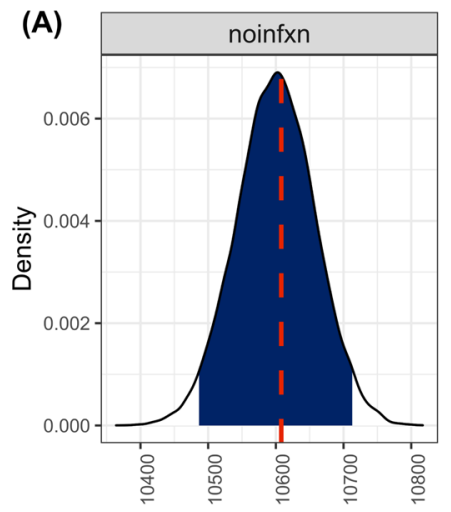

(B)

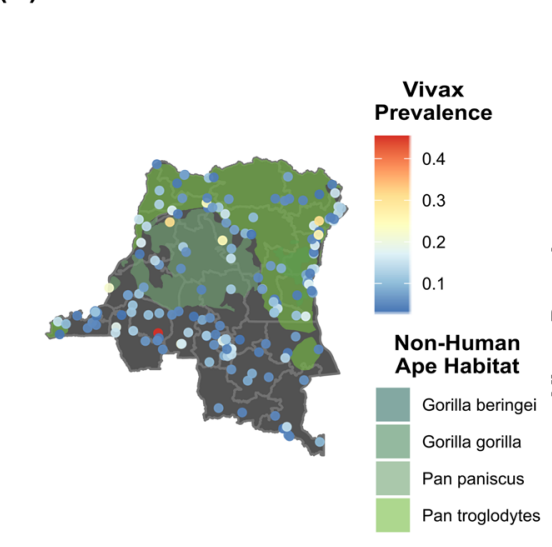

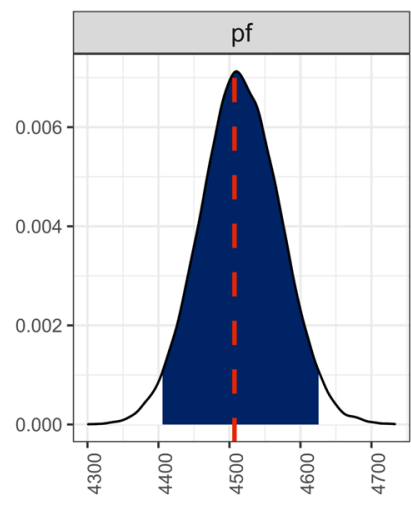

(C)
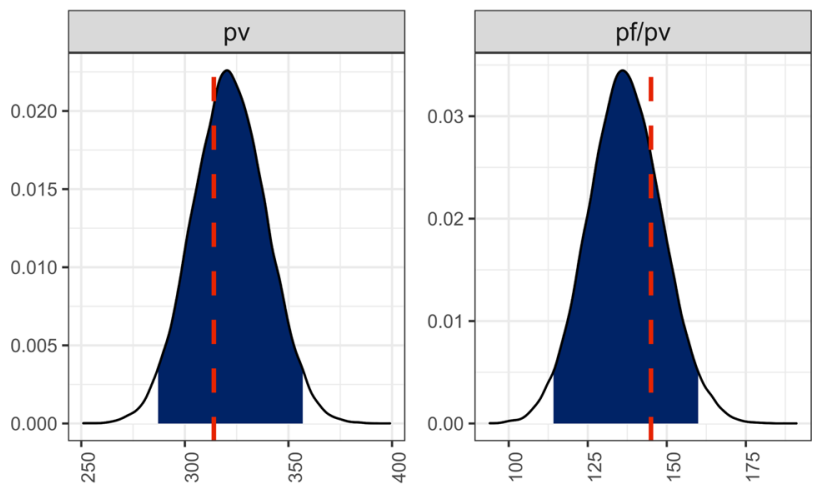

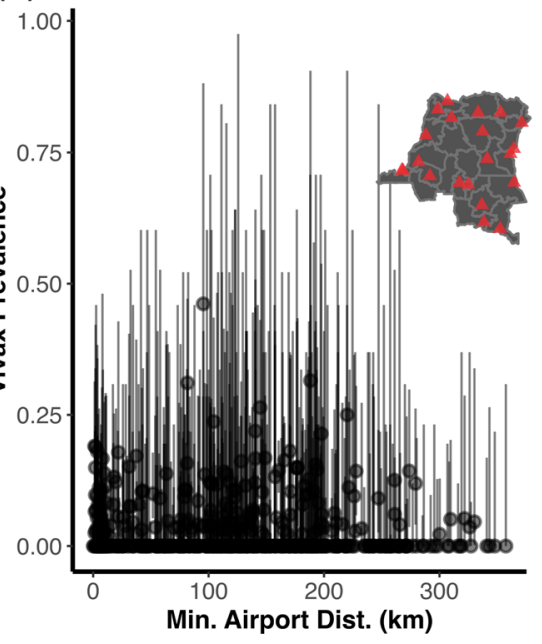

(D)

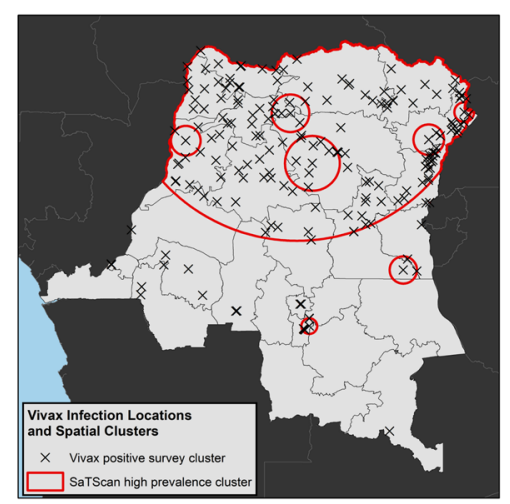

Fig. 3 Non-parametric risk factor analysis and spatial clustering of $\boldsymbol{P}$. vivax Infections. A Composition of $P$. vivax and $P$. falciparum co-infections. The expected versus observed composition of $P$. vivax and $P$. falciparum infections were explored using a multinomial probability likelihood model testing for independent acquisition of each species. To incorporate DHS sample weights, infections were rounded to the nearest integer. The plot shows the expected distribution for individuals without infection ("noinfxn"), P. falciparum infections ("pf"), P. vivax infections ("pv"), and P. falciparum-P. vivax co-infections ("pf/pv"). The blue shading indicates the $95 \%$ bootstrapped interval and the red-dotted line indicates the observed number of cases for each infection category. Overall, the observed distribution of each infection composition was explained well by each species occurring independently, which suggests that there were no interspecies infection synergism or antagonism. B Overlap with non-human ape habitats and positive P. vivax clusters. Overall, $P$. vivax prevalence did not appear to be associated with non-human ape habitat distribution (prevalence displayed as a proportion). This lack of a $P$. vivax-nonhuman ape association was recapitulated with permutation testing. Clusters with $P$. vivax infections are shaded on a blue-red spectrum with respect to the cluster-level prevalence, with the distribution of each non-human ape habitat is indicated in shades of green. C Correlation of $P$. vivax cluster prevalence with airport distance. The point estimate and $95 \%$ confidence interval for each cluster is shown as a point-range. Standard errors for the confidence intervals were calculated with the binomial exact method: to incorporate DHS weights, we rounded to the nearest integer. Airport distance was used as a proxy for the potentiality of $P$. vivax flight importation risk, or "airport malaria" risk. Overall, there did not appear to be a correlation between this variable and $P$. vivax prevalence (energy-correlation: 0.07). D Cluster detection of $P$. vivax infections. $P$. vivax infections appeared to be clustered in the northern and northeastern regions of the DRC with a small cluster also indicated in the center of the country.

of our three DRC $P$. vivax samples with publicly available isolates. Among the three sequenced DRC $P$. vivax samples that underwent next generation sequencing with hybrid selection enrichment, we achieved high-quality coverage in $\geq 98.0 \%$ of the mtDNA ( $5 \times$ coverage with $\mathrm{MQ} \geq 10, \mathrm{BQ} \geq 20$ ). Among the 655 out of 705 publicly available Illumina sequenced $P$. vivax globally sourced and DRC isolates that passed QC-thresholds, we identified 97 biallelic single nucleotide polymorphisms (SNPs) and 102 unique mitochondrial haplotypes. When removing conserved haplotypes within countries, we identified 142 country-unique mitochondrial haplotypes (N.B. some identical haplotypes are shared between countries; Supplementary Fig. 11). Among the global contemporary isolates, the $P$. vivax populations from China and Cambodia showed the greatest within-population mtDNA nucleotide diversity while within-population haplotype diversity appeared to be greatest in the population from Vietnam. Overall, there was limited within-population nucleotide and haplotype diversity among the isolates from the DRC (Supplementary Table 9).

Direct visualization of the mitochondrial consensus haplotypes indicated that the DRC consensus haplotype was identical to a consensus haplotype from Brazil ( $n=1$ isolate) and Thailand ( $n$ $=3$ isolates), as well as the Sall $P$. vivax lab strain consensus haplotype (base-pair difference range: 0-7). In contrast, the DRC mitochondrial consensus haplotype differed from Ebro-1944 and the non-human ape mitochondrial consensus haplotypes by three and four bases, respectively (Fig. 5A; Supplementary Fig. 11).

Considering Hamming's genetic distances, we found that the DRC samples shared a low level of relatedness with contemporary isolates from multiple regions, including both Asia and South 
(A)

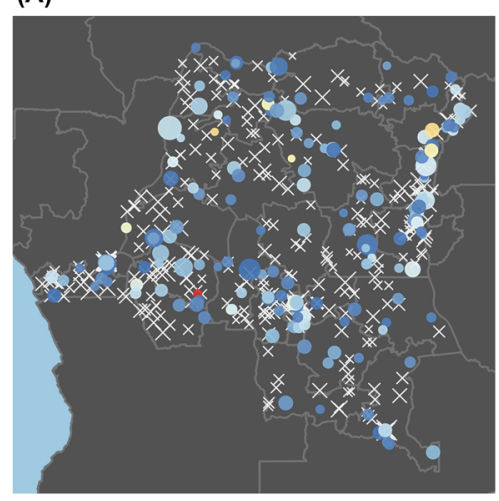

Prevalence
(B)

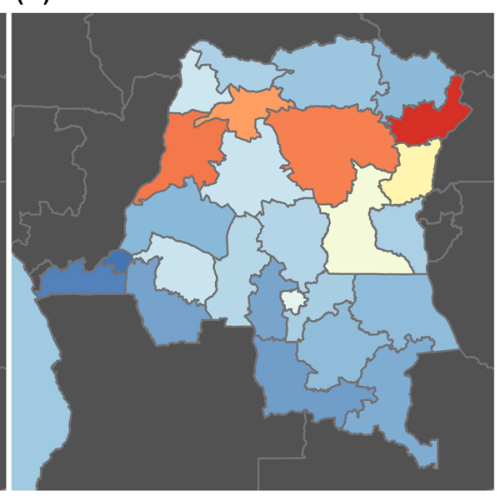

(C)

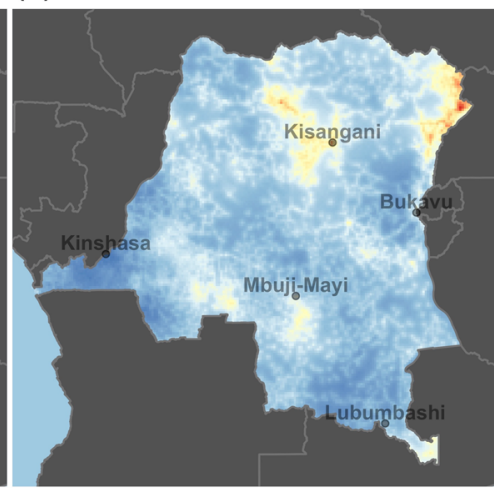
$0.0^{2} 0^{3} 0^{x}$

Prevalence

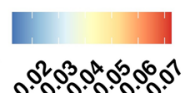

Prevalence

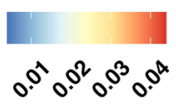

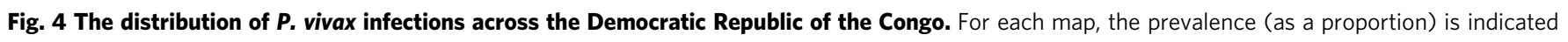

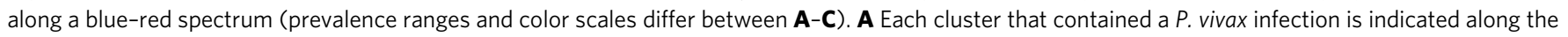

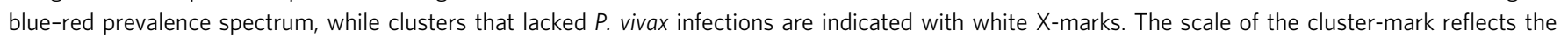
cluster sample size (weighted-range: 1.41-269.63). P. vivax infections appeared to be diffusely spread throughout the country with cluster prevalences ranging from 0 to $46.15 \%$. The cluster with the greatest prevalence, located in the Southeast near the Angola border, contained $6 / 13$ infections. However, when DHS sampling weights were considered, this cluster was shrunk considerably. In addition, several surrounding clusters had no infections, resulting in less evidence for a focal region of high prevalence on spatial modeling: as such, its point-prevalence should not be overinterpreted. B The posterior mean prevalence estimates for the province-level model, which show that $P$. vivax infections appeared to be slightly more common in the north and northeast. C The posterior mean prevalence estimates that were interpolated across the DRC from the cluster-level model indicated a diffuse, low-level of $P$. vivax prevalence across the country with a few regions of higher prevalence. These focal regions of higher prevalence appeared to be concentrated in the

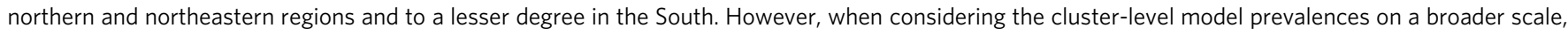
$\sim 99 \%$ of predicted prevalences are below the national average, which suggests a diffuse pattern of low prevalence across the DRC.

America (Fig. 5A). When considering a minimum-spanning network on a representative consensus haplotype from each country, we found that the DRC samples appeared to be most closely associated with contemporary $P$. vivax strains (Fig. 5B).

\section{Discussion}

$P$. vivax infections among adults in the $\mathrm{DRC}$ are more common than previously realized. From our spatially robust dataset, we detected 467 P. vivax infections corresponding to a DRC national prevalence of $2.96 \%$ (95\% $\left.\mathrm{CI}_{\text {weighted: }} 2.28,3.65 \%\right)$. Among those infected, nearly all were Duffy-negative (464/467, 99.36\%).

Risk-factors typically associated with $P$. vivax infection included precipitation and distance from healthcare facilities. The negative relationship between $P$. vivax prevalence and precipitation differed from other previous studies ${ }^{10,11}$, although the underlying effect is likely complicated by several ecological factors, such as temporal components, vector habitats, seasonality, altitude, and temperature ${ }^{12}$. Similarly, increased prevalence of falciparum malaria has previously been associated with access to healthcare resources in northern Ghana ${ }^{13}$ as well as other regions. These risk factors suggest that individuals that likely have less access to healthcare resources, particularly those who may be in climates that facilitate vivax malaria transmission, are more likely to be infected with $P$. vivax. However, the cluster-level spatial parameter estimate indicated that individuals further from healthcare facilities had lower associated $P$. vivax prevalences, possibly hinting at a complicated interaction between urbanicity and healthcare resources versus prevalence.

Overall, the $P$. vivax malaria risk factors differed from risk factors found for $P$. falciparum in this study using the same methodological approach. In addition, infection with $P$. falciparum did not seem to inhibit infection with $P$. vivax and viceversa. This contrast between $P$. vivax and $P$. falciparum risk factors may indicate different processes of transmission, with vivax-specific factors including: a shortened intrinsic period which may cause decreased efficacy of typical antimalarial interventions, hypnozoite infections resulting in relapse infections despite individual uptake of antimalarial strategies or behaviors, or different vector capacities ${ }^{14}$. For example, net use was not associated with a protective effect in $P$. vivax, which may reflect a hypnozoite reservoir or long-term carriage of parasites. As a result, despite being sympatric infections, $P$. falciparum and $P$. vivax risk factors may diverge due to differences in transmission, and therefore, may not be expected to overlap.

$P$. vivax infections were found throughout the entire country with a few focal regions of relatively high prevalence. The highest prevalence and clustering of $P$. vivax infections was found in north and northeastern regions, particularly in the Ituri province. This may be due to cross-border migration with South Sudan and Uganda, which are near countries that are endemic for $P$. vivax ( $P$. vivax infections have been reported in both countries $)^{5,7}$. In 2013, the United Nations noted that these regions had a large concentration of internally displaced persons and refugees, which qualitatively adds to this hypothesis ${ }^{15}$. Future $P$. vivax epidemiological studies in the DRC should consider collecting human mobility data (e.g. cell-phone data), particularly with respect to Kinshasa and regions along the northeastern border where interactions with Duffy-positive immigrants may be more frequent. With human mobility data, it may be possible to capture putative transmission chains between Duffy-positive immigrants and DRC Duffy-negative inhabitants that would help to characterize the extent of secondary transmission and potential forward propagation of disease.

The sources of infection in other regions in the DRC was less clear, where most prevalence estimates ranged from $\sim 0$ to $2 \%$. The scattering of infections across most of the DRC, as indicated by the maps and more than one-third of all $P$. vivax infected 
(A)

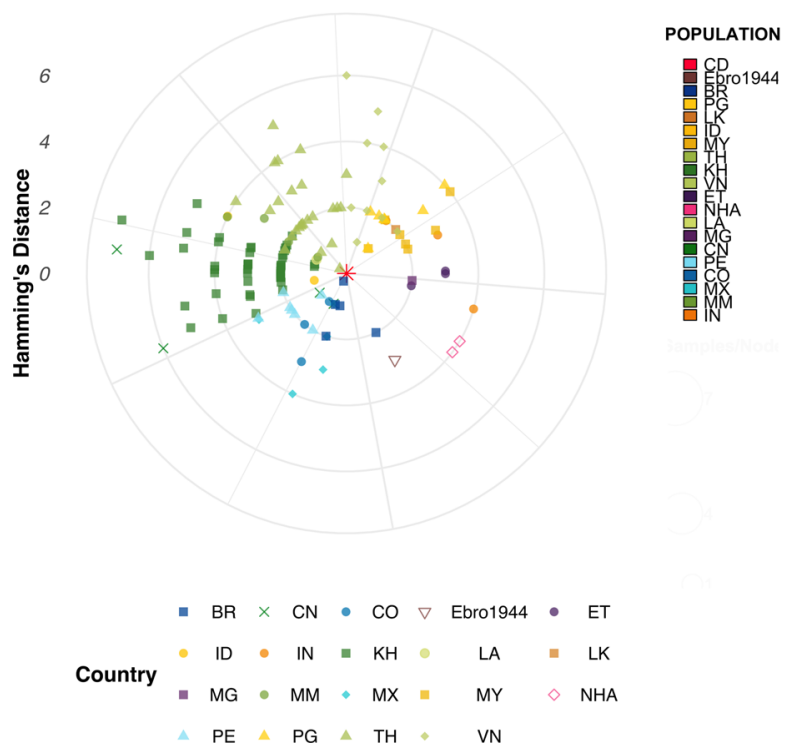

(B)

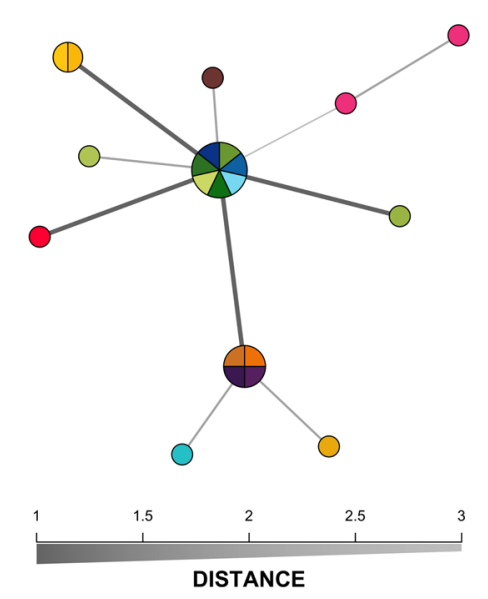

Fig. 5 P. vivax mitochondrial Hamming's distances and minimum-spanning network. Comparison of DRC $P$. vivax with over 700 globally sourced $P$. vivax isolates yielded 142 within-country unique consensus haplotypes and 102 overall unique consensus haplotypes, as duplicates existed within countries. In the instance of the DRC consensus haplotypes $(n=2 / 3)$, one isolate was identical except for ten missing sites. As a result, the two DRC consensus haplotypes were reduced to a single sequence and used in the above figures. A The DRC consensus haplotype is depicted in the center (red asterisk) and orbited by the other 140 consensus haplotypes. Distances from the center are determined by the number of base-pair differences while the relative position along the orbit is arbitrary and clustered only for visualization aid. Isolates from the Americas are colored in shades of blue and include Brazil (BR), Colombia (CO), Mexico (MX), and Peru (PE) Isolates from Asia are indicated in shades of yellow-green and include China (CN), Indonesia (ID), Cambodia $(\mathrm{KH})$, Laos (LA), Myanmar (MM), Malaysia (MY), Papua New Guinea (PG), Thailand (TH), and Vietnam (VN). India (IN) and Sri Lanka (LK) are indicated in shades of orange, while Ethiopia (ET) and Madagascar (MG) are indicated in shades of purple. Finally, the Democratic Republic of the Congo (DRC) is shown in red and non-human apes (NHA) are shown in magenta. The historical sample from the Ebro Delta in Spain dating to 1944 (Ebro1944) is colored in brown. B A representative consensus haplotype from each country and both unique non-human ape sequences were selected as input into the minimum-spanning tree. Overall, there are similarities among the haplotypes from both the Americas and Asia, as well as the DRC consensus haplotype, although to a lesser degree. In terms of base-pair differences (Hamming's distance), the DRC mitochondrial consensus haplotype differed by three and four bases to the Ebro1944 and the non-human ape consensus haplotypes, respectively. All unique consensus haplotypes with respect to country of origin are provided for comparison and context (Supplementary Fig. 11).

clusters containing only a single infection, suggests that $P$. vivax is diffusely distributed at a relatively low prevalence across most of the DRC. This essentially flat distribution of vivax malaria across the DRC contrasts the broad spatial distribution of $P$. falciparum infections previously observed in the 2007 and 2013 DRC DHS $^{16,17}$. As a result, we suggest that $P$. vivax has been unable to gain a foothold in the region and is persisting rather than breaking out.

The relatively large differences in our DRC $P$. vivax and the non-human ape mitochondrial genomes negates recent zoonotic transmission as the source of DRC $P$. vivax ${ }^{18}$. This conclusion is strengthened by the lack of a significant relationship between $P$. vivax prevalence and non-human ape habitats. Similarly, we found that the now extinct European $P$. vivax strain, taken from a historical sample originating from the Ebro Delta in Spain, circa $1944^{19,20}$, differed by several bases from our DRC P. vivax consensus haplotypes. Instead, our DRC mitochondrial consensus haplotype was identical to consensus haplotypes found in Brazil, Thailand, and the Sal1 lab strain. This finding suggests that the DRC $P$. vivax strains fall within the mitochondrial diversity observed in contemporary $P$. vivax strains, although low levels of diversity and geographic structure precluded assessment using phylogenetic approaches. While the exact phylogeographic affiliations of these DRC P. vivax isolates is challenging to pinpoint with these mitochondrial genomes alone, we provide strong evidence that these infections did not originate from non-human apes and are likely distinct from the extinct European $P$. vivax circulating in the 1940s.

Although the mitochondrial genome is a non-recombining region with putatively neutral SNPs that is ideal for phylogenetic analysis, the few number of segregating sites precluded assessment of fine-scale geographic affiliations from the mtDNA. This relative lack of informative sites is consistent with previous reports ${ }^{21}$, but may also be an artifact of our small sample sizes in specific locations or our conservative approach to variant filtering. In addition, among the DRC mtDNA sequences, the variant at site 5910 was originally heterozygous and should be considered a low-confidence SNP. Future work with additional biological material to improve the likelihood of successful genomic sequencing will be needed to capture more genomic information allowing the application of more sophisticated methodological approaches such as coalescent modeling and spatio-demographic inference. Determining if DRC $P$. vivax has been recently imported, or is a distinct clade that has resulted from long-standing, neglected endemic transmission-as suggested in Mauritania ${ }^{22}$-will be informative for policymakers to determine the feasibility and urgency of vivax control and elimination efforts. In addition, with $P$. vivax whole genomes, signatures of selection for adaptation to the Duffynegative host can be evaluated, which would help to better characterize and predict the threat of Duffy-negative transmission and disease. 
The main limitations of our study are the cross-sectional design, which limits inference of effects with a temporal component (e.g. seasonality), the DHS sampling design, which restricts the study population largely to asymptomatic individuals and misses critical age groups ${ }^{23}$, the proxies and spatial resolution of the various risk factors, and the aforementioned small number of high-quality DRC sequences generated. In addition, the $\Phi$ parameter and the $\sigma^{2}$-parameter in the cluster-level spatial model, as well as the $\rho$-parameter in the province-level spatial model, remained somewhat unstable, potentially reflecting the complicated spatial autocorrelation among the $P$. vivax prevalences. Similarly, although limits of power were explored, it is possible that $P$. vivax specific risk factors were missed given the relatively few infections and wide confidence intervals. A spatially robust, large prospective cohort study across all ages and across multiple seasons could address many of these limitations, but costs are likely to be prohibitive.

Until recently, $P$. vivax, was an unrecognized cause of disease in SSA. The DRC is a critical region for the study of malaria in SSA due to its geographic size, central location, and the evidence that it bridges East and West Africa malaria ${ }^{8,9}$. Although previous studies have screened large populations in SSA for P. vivax ${ }^{6,24-28}$, this study provides a systematic and nationally representative survey of $P$. vivax in a SSA country not considered endemic for the disease. We demonstrated that $P$. vivax is circulating at prevalences higher than previously thought, despite a high frequency of Duffy-negativity ${ }^{7}$. However, $P$. vivax infections were associated with few risk factors, were spread diffusely throughout the country, and did not have a clear genetic ancestry based on the mitochondrial genome, with the exception that these are likely not zoonotic infections from non-human apes and likely not an ancestral remnant ${ }^{18-21}$. Instead, the DRC $P$. vivax strains appear to be contemporary, prompting three possible and non-mutually exclusive explanations: (1) these infections are frequently present at sub-microscopic or low parasitemia that potentially limit transmissibility ${ }^{29},(2)$ these infections are the result of continual importation of $P$. vivax with limited forward-propagation, or (3) infections are the result of long-standing relapse as primaquine is not routinely administered in SSA. Although there are numerous other explanations, all three of these explanations are consistent with previous work that suggests $P$. vivax infections among Duffy-negative individuals are frequently mild and asymptomatic compared with Duffy-positive individuals ${ }^{5,6}$. Finally, emerging research suggests that genotypically Duffy-negative hosts express the Duffy antigen among erythroid progenitors in the bone marrow and that $P$. vivax gametocytes are able to mature and proliferate in the bone marrow of non-human primate animal models $^{30,31}$. Collectively, this suggests that $P$. vivax in SSA may be persisting as low parasitemic, asymptomatic infections, by hiding in the bone marrow or other tissues containing early progenitor red blood cells of Duffy-negative hosts ${ }^{30-32}$. As a result, if these infections are typically asymptomatic and present with lower parasitemia that limit transmissibility, they pose a limited morbidity cost to the individual and an overall low public health threat.

P. vivax infections among Duffy-negative individuals appear to be occurring throughout SSA $6,24-28$. However, the current distribution and low prevalence of vivax malaria in SSA supports continued investments targeting $P$. falciparum as likely having the greatest impact on malaria control, morbidity, and mortality. Future efforts targeted at the DRC malaria elimination end-game may need to consider vivax-specific interventions.

\section{Methods}

Study participants and malaria detection. We studied men and women aged 15-59 years and 15-49 years, respectively, that were surveyed in the 2013-2014
DRC DHS. Each participant answered an extensive questionnaire and provided a dried blood spot (DBS) for HIV and other biomarker screening. Spatial and ecological data were collected for each sampling cluster (Supplementary Materials: Covariate Feature Engineering, Spatial and Raster Feature Engineering). We extracted DNA from each DBS using Chelex-100 (Bio-Rad, Hercules, CA) and Saponin and then screened all participants for $P$. vivax using qPCR targeting the 18 S ribosomal RNA gene ${ }^{33}$. Samples that screened positive by $18 \mathrm{~S}-\mathrm{qPCR}$ underwent reflex confirmatory screening using a nested-PCR assay targeting $18 \mathrm{~S}$ rRNA (Supplementary Table 1$)^{34}$. To ensure the quality of DNA extraction, we excluded samples that had previously failed to amplify human-beta-tubulin ${ }^{17}$ from analysis. Finally, participants were excluded if they had missing data or were not a part of the DHS sampling schematic (Fig. 1) ${ }^{35}$. This study reanalyzes previously published $P$. falciparum data that was generated with a $P$. falciparum lactate dehydrogenase gene qPCR approach (sample size differences are due to different inclusion criteria) ${ }^{17}$. This study was approved by the IRBs at the University of North Carolina at Chapel Hill and the Kinshasa School of Public Health.

Duffy genotyping. All samples that initially screened positive underwent Host Duffy antigen/chemokine receptor (DARC) genotyping. The DARC genotype was determined using a previously validated High-Resolution Melt (HRM) assay (Supplementary Table 3$)^{36}$. Genotypes that could not be definitely resolved by HRM were reconciled by Sanger sequencing ${ }^{6}$. In addition, HRM results were validated by sequencing $\sim 10 \%$ samples (Supplementary Materials: DuffyGenotyping).

Risk factor modeling. $P$. vivax risk factors were identified from a comprehensive literature search and previous work from the 2013 to 2014 DRC DHS identifying $P$. falciparum risk factors ${ }^{13,16,17,37}$. Risk factors were derived from the DHS questionnaires and other open-data sources (Supplementary Materials: Covariate Feature Engineering) https://www.hotosm.org/35,38-41. All continuous risk factors were standardized in order to promote model stability and ease of comparability For dichotomized risk factors, the a priori protective level was selected as the referent level (e.g. HIV-negative) or the largest group if a protective level was not obvious (e.g. female for biological sex).

For each risk factor, confounding covariates were identified using a directed acyclic diagram (DAG) built from our a priori causal framework of covariate and outcome relationships (Supplementary Fig. 3). To confirm manageable collinearity, we analyzed the energy correlation between all covariates (Supplementary Fig. 4). We then used IPW to obtain marginal structural models and account for confounding between our risk factors and outcome of interest, malaria ${ }^{42-45}$. IPWs were calculated with a super learner algorithm, which uses a loss-based approach with $\mathrm{V}$-fold cross-validation to maximize predictions from an ensemble of candidate algorithms (Supplementary Table 4$)^{46}$. We extended the standard super learner algorithm to account for spatial dependence among observations using spatial cross validation (Supplementary Materials: Inverse Probability Weights and Prevalence Odds Ratios; Supplementary Fig. 5) ${ }^{47}$. The super learner algorithm was originally selected for IPW calculations to account for known issues and biases of functional form in fitting the exposure dose-response curve ${ }^{48}$. However, as stated above, the super learner candidate library was reduced to a regression model in 9/ 11 models in favor of convergence or better IPW stability. IPWs and DHS sampling weights were accounted for under the assumption that the distribution of the sampling was independent of the distribution of confounding covariates, which allows for weights to be considered jointly, $w_{f}=w_{s}{ }^{*} w_{i p w}$. We then used the 'survey' $\mathrm{R}$ package to account for the DHS sampling design and to perform the generalized estimating equation (GEE) regression. The GEEs were calculated with a logit-link function and binomial variance to produce odds ratios. Finally, we performed power calculations given our study and sample characteristics to determine the extent of the power that we had to detect significant odds ratios (Supplementary Materials: post-hoc Power Calculations)

In addition, we considered several alternative $P$. vivax risk factors that could not be estimated with a parametric approach due to model assumption violations or a lack of data. These additional risk factors included: (1) the putative Duffy phenotype (Supplementary Materials: Duffy-Genotyping); (2) within-host interactions of $P$. vivax and $P$. falciparum using a multinomial likelihood-based model that assumes independent infection acquisition (Supplementary Materials: $P$. falciparum-P. vivax Co-infection Model $)^{49}$; (3) interactions between nonhuman ape ranges and $P$. vivax cluster-level prevalences using permutation tests (Supplementary Materials: Overlap with Non-Human Ape Permutation Testing) ${ }^{18,50}$; and (4) the association between $P$. vivax cluster prevalence and the proximity to airports, as a proxy for importation of $P$. vivax via airline travel. Proximity to airports was calculated as the minimum greater-circle distance from each cluster to an airport that was classified as "medium" or "large" (Supplementary Materials: Covariate Feature Engineering).

P. vivax spatial analyses and prevalence mapping. Spatial clustering of $P$. vivax was initially assessed using spatial scan statistics through the 'SaTScan' (v9.6.1) platform ${ }^{51}$. A Poisson distribution of cases was assumed and the model was specified to detect only clusters of higher prevalence relative to neighboring survey cluster locations. A significance threshold of 0.05 was set for cluster detection. 
We considered spatial autocorrelation with Moran's I using a province adjacency matrix as well as a matrix of greater-circle distances between clusters ${ }^{52}$ Greater-circle distances were calculated using a geodesic approach ${ }^{53}$. Significance was evaluated using a permutation test with 100,000 iterations and a one-sided $p$ value.

To determine the spatial distribution of $P$. vivax, we fit two types of Bayesian mixed spatial models: (1) a province-level areal model and (2) a cluster-level Gaussian spatial process model ${ }^{54,55}$. Both sets of spatial models were fit with generalized linear mixed models using the logistic link function and a binomial error distribution with a spatial random effect. The selected priors and the full model formulations are available in the Supplementary Materials: Bayesian Spatial Prevalence Models. For each of the respective spatial-levels, we used the identified significant risk factors in the model fitting. Spatial covariates were extracted from the Climate Hazards Group Infrared Precipitation with Stations (precipitation) ${ }^{39}$ using the 'environmentalinformatics-marburg/heavyRain' wrapper and the Malaria Atlas Project (average walking travel times to health care facilities) ${ }^{41}$ to incorporate the significant risk factors that were identified (Supplementary Materials: Spatial and Raster Feature Engineering).

For simplicity, we assumed the WGS84 projection system throughout this analysis, which includes all risk factors with a spatial component, spatial covariates, and spatial models. This assumption is relatively minor as the DRC straddles the equator.

P. vivax mitochondrial genomics. DNA quantity and quality was limited from the adult participants considered in this study taken from the 2013 to 2014 DRC DHS. However, DNA from children collected as part of the 2013-2014 DRC DHS was available at a higher quality and was able to be sequenced ${ }^{56}$. From this previous study, DNA from three previously identified samples was successfully amplified using the Illustra Genomic Phi V2 DNA Amplification Kit (GE Healthcare Life Sciences, Pittsburgh, PA) and sequencing libraries prepared with NEBNext Ultra DNA Library Prep Kit for Illumina (New England BioLabs Inc., Ipswich, MA). Amplified libraries were then enriched using a custom in-solution product (MYbaits) targeting the P. vivax genome (version 3.0; MYcroarray: The Oligo Library Company, Ann Arbor, MI). Enriched genomes were sequenced on MiSeq 150 base-pair paired-end and HiSeq2500 125 base-pair paired-end chemistry (Illumina, San Diego, CA) platforms. All subsequent analyses were limited to the mtDNA due to low coverage in the nuclear genome. Nucleotide variants were identified among all samples and unique consensus haplotypes were determined (Supplementary Materials: Variant Filtering and Consensus Haplotypes). Using these three DRC samples and the globally sourced mitochondrial alignments, we calculated basic population genetic statistic summaries, including: withinpopulation nucleotide diversity, counts of unique consensus haplotypes, and between-sample Hamming's distance (i.e. base-pair differences). For our basic genetic distance analysis, we removed duplicate consensus haplotypes within countries and visualized base-pair differences (Supplementary Materials: Population Genetic Statistics and Distances). Next, we subset the data to a representative consensus haplotype for each country and created a minimum-spanning network (Supplementary Materials: Population Genetic Statistics and Distances).

Reporting summary. Further information on research design is available in the Nature Research Reporting Summary linked to this article.

\section{Data availability}

Due to data privacy considerations relating to the Demographic Health Survey, the molecular PCR results generated and analyzed during the current study are available from the corresponding author upon reasonable request. In addition, all epidemiological covariate data is available upon reasonable to the corresponding author, including intermediary files. The genomic data produced by the current study is available at the short reads archive bioproject: PRJNA725254.

\section{Code availability}

All scripts and code used to generate these analyses are publicly available on Github (Epidemiological Analyses: github.com/nickbrazeau/VivID_Epi; Population Genetic Analyses: github.com/nickbrazeau/VivID_Seq). The custom scripts used in this analysis are available under the Github repositories: github.com/nickbrazeau/mlrwrapSL, github. com/IDEELResearch/vcfRmanip, github.com/nickbrazeau/icer.

Received: 25 March 2020; Accepted: 1 June 2021; Published online: 07 July 2021

\section{References}

1. WHO Team: Global Malaria Programme. World Malaria Report 2020. https:// www.who.int/publications/i/item/9789240015791 (2020).
2. Miller, L. H., Mason, S. J. \& Clyde, D. F. The resistance factor to Plasmodium vivax in blacks: the Duffy-blood-group genotype, FyFy. N. Engl. J. 295, 302-4(1976).

3. Tournamille, C., Colin, Y. \& Cartron, J. P. \& Van Kim, C. L. Disruption of a GATA motif in the Duffy gene promoter abolishes erythroid gene expression in Duffy-negative individuals. Nat. Genet. 10, 224-228 (1995).

4. Howes, R. E. et al. The global distribution of the Duffy blood group. Nat. Commun. 2, 266 (2011).

5. Twohig, K. A. et al. Growing evidence of Plasmodium vivax across malariaendemic Africa. PLoS Negl. Trop. Dis. 13, e0007140 (2019).

6. Ménard, D. et al. Plasmodium vivax clinical malaria is commonly observed in Duffy-negative Malagasy people. Proc. Natl Acad. Sci. USA. 107, 5967-5971 (2010).

7. Battle, K. E. et al. Mapping the global endemicity and clinical burden of Plasmodium vivax, 2000-17: a spatial and temporal modelling study. Lancet 394, 332-343 (2019).

8. Verity, R. J., Aydemir, O., Brazeau, N. F. \& Watson, O. J. The impact of antimalarial resistance on the genetic structure of Plasmodium falciparum in the DRC. bioRxiv 11, 2107. https://www.nature.com/articles/s41467-02015779 (2020).

9. Taylor, S. M. et al. Plasmodium falciparum sulfadoxine resistance is geographically and genetically clustered within the DR Congo. Sci. Rep. 3, 1165 (2013).

10. Bi, Y. et al. Impact of climate variability on Plasmodium vivax and Plasmodium falciparum malaria in Yunnan Province, China. Parasit Vectors. 6, 357 (2013).

11. Kim, Y.-M., Park, J.-W. \& Cheong, H.-K. Estimated effect of climatic variables on the transmission of Plasmodium vivax malaria in the Republic of Korea. Environ. Health Perspect. 120, 1314-1319 (2012).

12. Chowell, G., Munayco, C. V., Escalante, A. A. \& McKenzie, F. E. The spatial and temporal patterns of falciparum and vivax malaria in Perú: 1994-2006. Malar. J. 8, 142 (2009).

13. Millar, J. et al. Detecting local risk factors for residual malaria in northern Ghana using Bayesian model averaging. Malar. J. 17, 343 (2018).

14. Olliaro, P. L. et al. Implications of Plasmodium vivax Biology for Control, Elimination, and Research. Am J. Trop. Med. Hyg. 95, 4-14 (2016).

15. United Nations High Commissioner for Refugees. UNHCR D.R.Congo Fact Sheet. https://data2.unhcr.org/en/documents/download/48441 (2013).

16. Taylor, S. M. et al. Molecular Malaria Epidemiology: Mapping and Burden Estimates for the Democratic Republic of the Congo, 2007. PLoS One 6, e16420 (2011).

17. Deutsch-Feldman, M. et al. Spatial and epidemiological drivers of $P$. falciparum malaria among adults in the Democratic Republic of the Congo. BMJ Global Health (2020) https://doi.org/10.1136/bmjgh-2020-002316.

18. Liu, W. et al. African origin of the malaria parasite Plasmodium vivax. Nat. Commun. 5, 3346 (2014).

19. Gelabert, P. et al. Mitochondrial DNA from the eradicated European Plasmodium vivax and P. falciparum from 70-year-old slides from the Ebro Delta in Spain. Proc. Natl Acad. Sci. USA 113, 11495-11500 (2016).

20. van Dorp, L., Gelabert, P., Rieux, A. \& de Manuel, M. Plasmodium vivax Malaria viewed through the lens of an eradicated European strain. bioRxiv 37, 773-785. https://academic.oup.com/mbe/article/37/3/773/5614438 (2020).

21. Rodrigues, P. T. et al. Human migration and the spread of malaria parasites to the New World. Sci. Rep. 8, 1993 (2018).

22. $\mathrm{Ba}, \mathrm{H}$. et al. Multi-locus genotyping reveals established endemicity of a geographically distinct Plasmodium vivax population in Mauritania, West Africa. PLoS Negl. Trop. Dis. 14, e0008945 (2020).

23. Deutsch-Feldman, M. et al. What is the burden of malaria in the DRC? J. Infect. Dis. https://doi.org/10.1093/infdis/jiaa650 (2020).

24. Culleton, R. L. et al. Failure to detect Plasmodium vivax in West and Central Africa by PCR species typing. Malar. J. 7, 174 (2008).

25. Mendes, C. et al. Duffy negative antigen is no longer a barrier to Plasmodium vivax-molecular evidences from the African West Coast (Angola and Equatorial Guinea). PLoS Negl. Trop. Dis. 5, e1192 (2011).

26. Poirier, P. et al. The hide and seek of Plasmodium vivax in West Africa: report from a large-scale study in Beninese asymptomatic subjects. Malar. J. 15, 570 (2016).

27. Motshoge, T. et al. Molecular evidence of high rates of asymptomatic $P$. vivax infection and very low P. falciparum malaria in Botswana. BMC Infect. Dis. 16, 520 (2016).

28. Woldearegai, T. G., Kremsner, P. G., Kun, J. F. J. \& Mordmüller, B. Plasmodium vivax malaria in Duffy-negative individuals from Ethiopia. Trans. R. Soc. Trop. Med. Hyg. 107, 328-331 (2013).

29. Koepfli, C. et al. Blood-Stage Parasitaemia and age determine Plasmodium falciparum and P. vivax Gametocytaemia in Papua New Guinea. PLoS One 10, e0126747 (2015).

30. Dechavanne, C. et al. Duffy Antigen Expression in Erythroid Bone Marrow Precursor Cells of Genotypically Duffy Negative Individuals. bioRxiv 508481. https://doi.org/10.1101/508481 (2018). 
31. Obaldia, N., 3rd et al. Bone Marrow Is a Major Parasite Reservoir in Plasmodium vivax Infection. MBio 9, e00625-18 (2018).

32. Mumau, M. D. et al. Identification of a multipotent progenitor population in the spleen that is regulated by NR4A1. J. Immunol. 200, 1078-1087 (2018).

33. Srisutham, S. et al. Four human Plasmodium species quantification using droplet digital PCR. PLoS One 12, e0175771 (2017).

34. Snounou, G. \& Singh, B. Nested PCR analysis of Plasmodium parasites. Methods Mol. Med. 72, 189-203 (2002).

35. Croft, T. N., Marshall, A. M. J., Allen, C. K. \& Others. Guide to DHS statistics. (ICF, 2018).

36. Tanaka, M., Takahahi, J., Hirayama, F. \& Tani, Y. High-resolution melting analysis for genotyping Duffy, Kidd and Diego blood group antigens. Leg. Med. 13, 1-6 (2011).

37. Tusting, L. S. et al. Housing Improvements and Malaria Risk in Sub-Saharan Africa: A Multi-Country Analysis of Survey Data. PLoS Med. 14, e1002234 (2017).

38. API Client and Dataset Management for the Demographic and Health Survey (DHS) Data [R package rdhs version 0.6.3].

39. Funk, C. et al. The climate hazards infrared precipitation with stations-a new environmental record for monitoring extremes. Sci. Data 2, 150066 (2015).

40. Garske, T., Ferguson, N. M. \& Ghani, A. C. Estimating air temperature and its influence on malaria transmission across Africa. PLoS One 8, e56487 (2013).

41. Weiss, D. J. et al. Global maps of travel time to healthcare facilities. Nat. Med. 26, 1835-1838 (2020).

42. Hernán M. A., R. J. M. Causal Inference. (Chapman \& Hall/CRC).

43. Hernán, M. A. \& Robins, J. M. Estimating causal effects from epidemiological data. J. Epidemiol. Community Health 60, 578-586 (2006).

44. Robins, J. M., Hernán, M. A. \& Brumback, B. Marginal structural models and causal inference in epidemiology. Epidemiology 11, 550-560 (2000).

45. Cole, S. R. \& Hernán, M. A. Constructing inverse probability weights for marginal structural models. Am. J. Epidemiol. 168, 656-664 (2008).

46. van der Laan, M. J., Polley, E. C. \& Hubbard, A. E. Super learner. Stat. Appl. Genet. Mol. Biol. 6, Article25 (2007).

47. Brenning, A. Spatial cross-validation and bootstrap for the assessment of prediction rules in remote sensing: The R package sperrorest. in 2012 IEEE International Geoscience and Remote Sensing Symposium 5372-5375 (2012).

48. Pirracchio, R., Petersen, M. L. \& van der Laan, M. Improving propensity score estimators' robustness to model misspecification using super learner. Am. J. Epidemiol. 181, 108-119 (2015).

49. Akala, H. M. et al. Longitudinal characterization of Plasmodium inter-species interactions during a period of increasing prevalence of Plasmodium ovale. medRxiv. https://doi.org/10.1101/2019.12.28.19015941 (2020).

50. Liu, W. et al. Wild bonobos host geographically restricted malaria parasites including a putative new Laverania species. Nat. Commun. 8, 1635 (2017).

51. Kulldorff, M. A spatial scan statistic. Commun. Stat. - Theory Methods 26, 1481-1496 (1997).

52. Moran, P. A. P. Notes on continuous stochastic phenomena. Biometrika 37, 17-23 (1950).

53. Karney, C. F. F. Algorithms for geodesics. J. Geod. 87, 43-55 (2013).

54. Lee, D. CARBayes version 4.6: An R Package for Spatial Areal Unit Modelling with Conditional Autoregressive Priors. (University of Glasgow, 2017).

55. Giorgi, E., Diggle, P. J. \& Others. PrevMap: an R package for prevalence mapping. J. Off. Stat. 78, 2642 (2017).

56. Brazeau N. F., et al Plasmodium vivax Infections in Duffy-Negative Individuals in the Democratic Republic of the Congo. Am. J. Trop. Med. Hyg. https://doi.org/10.4269/ajtmh.18-0277 (2018).

\section{Acknowledgements}

The authors would like to thank the congolese survey field teams and study participants. The authors also thank the numerous open source data platforms that were used in this study, including the Demographic Health Survey from the Democratic Republic of the Congo 2013-2014, Open Street Map (๔ OpenStreetMap contributors: https://www. openstreetmap.org/copyright), Database of Global Administrative Areas, Level-1 and Atmosphere Archive \& Distribution System Distributed Active Archive Center, and the Earth Observations Group at National Oceanic. We also thank the European Nucleotide Archive and previous authors for making their next generation sequencing data available to the public. Finally, the authors would also like to thank Sandra Mendoza Guerrero for help with the hybrid captures. This research was funded by the National Institutes of Health: F30AI143172 (N.F.B.), R01TW010870 (J.J.J.), K24AI13499 (J.J.J.), R01AI107949 (S.R.M.), F30MH103925 (A.P.M.), T32AI070114 (M.D.F.) and the Wellcome Trust: 109312/Z/15/Z (O.J.W.).

\section{Author contributions}

N.F.B. designed experiments, conducted laboratory work, conducted analyses, and wrote the manuscript. C.L.M. conducted laboratory work, advised on analyses, and participated in manuscript preparation. A.P.M., O.J.W., M.D.F., V.G., B.R., J.E., R.V., C.K., helped develop software, advised on analyses, and participated in manuscript preparation. M.K. M., A.K.T., J.L.L., collected samples and participated in manuscript preparation. P.G. and L.v.D. provided data, advised on analyses, and participated in manuscript preparation. A.W. and K.L.T. conducted laboratory work and participated in manuscript preparation. M.E., G.W., J.B.P., S.R.M., and J.J.J. helped conceive the study, contributed to the experimental design, advised on analyses, and participated in manuscript preparation.

\section{Competing interests}

The authors declare no competing interests.

\section{Additional information}

Supplementary information The online version contains supplementary material available at https://doi.org/10.1038/s41467-021-24216-3.

Correspondence and requests for materials should be addressed to N.F.B

Peer review information Nature Communications thanks Katherine Battle, Jane Carlton, and Gillian Stresman for their contribution to the peer review of this work.

Reprints and permission information is available at http://www.nature.com/reprints

Publisher's note Springer Nature remains neutral with regard to jurisdictional claims in published maps and institutional affiliations.

Open Access This article is licensed under a Creative Commons Attribution 4.0 International License, which permits use, sharing, adaptation, distribution and reproduction in any medium or format, as long as you give appropriate credit to the original author(s) and the source, provide a link to the Creative Commons license, and indicate if changes were made. The images or other third party material in this article are included in the article's Creative Commons license, unless indicated otherwise in a credit line to the material. If material is not included in the article's Creative Commons license and your intended use is not permitted by statutory regulation or exceeds the permitted use, you will need to obtain permission directly from the copyright holder. To view a copy of this license, visit http://creativecommons.org/ licenses/by/4.0/.

(C) The Author(s) 2021 